\title{
enzyme-reaction chip
}

\author{
Qiannan Duan, ${ }^{\text {a, b }}$ Jianchao Lee,,${ }^{\text {a * }}$ Jinhong Gao, ${ }^{\mathrm{a}}$ Jiayuan Chen, ${ }^{\mathrm{a}}$ Yachao Lian, ${ }^{\mathrm{a}}$ Zoudi Wang, ${ }^{\mathrm{c}}$ Can Wang, ${ }^{\mathrm{d}}$ \\ Zhaoyi Xu, ${ }^{\mathrm{b}}$ Juan Ren, ${ }^{\mathrm{c}}$ Sifan $\mathrm{Bi}^{\mathrm{a}}$ \\ ${ }^{a}$ Department of Environment Science, Shaanxi Normal University, Xi'an 710062, China \\ ${ }^{\mathrm{b}}$ State Key Laboratory of Pollution Control and Resource Reuse, School of the Environment, Nanjing \\ University, Nanjing 210046, China \\ ${ }^{c}$ Shaanxi SIER Biotechnology co. LTD, Dushizhimen D Building, Jinye Road, High-tech Zone, Xi'an, \\ Shaanxi Province, 710077 \\ ${ }^{d}$ Big Data and Urban Spatial Analytics Laboratory, Tongji University, Shanghai 200092, China
}

\begin{abstract}
Machine learning (ML) has brought significant technological innovations in many fields, but it has not been widely embraced by most researchers of natural sciences to date. Traditional understanding and promotion of chemical analysis cannot meet the definition and requirement of big data for running of ML. Over the years, we focused on building a more versatile and low-cost approach to the acquisition of copious amounts of data containing in a chemical reaction. The generated data meet exclusively the thirst of ML when swimming in the vast space of chemical effect. As proof in this study, we carried out a case for acute toxicity test throughout the whole routine, from model building, chip preparation, data collection, and ML training. Such a strategy will probably play an important role in connecting ML with much research in natural science in the future.
\end{abstract}

Keyword: machine learning, toxicity test, high-throughput experimentation, inkjet printing, enzyme reaction chips
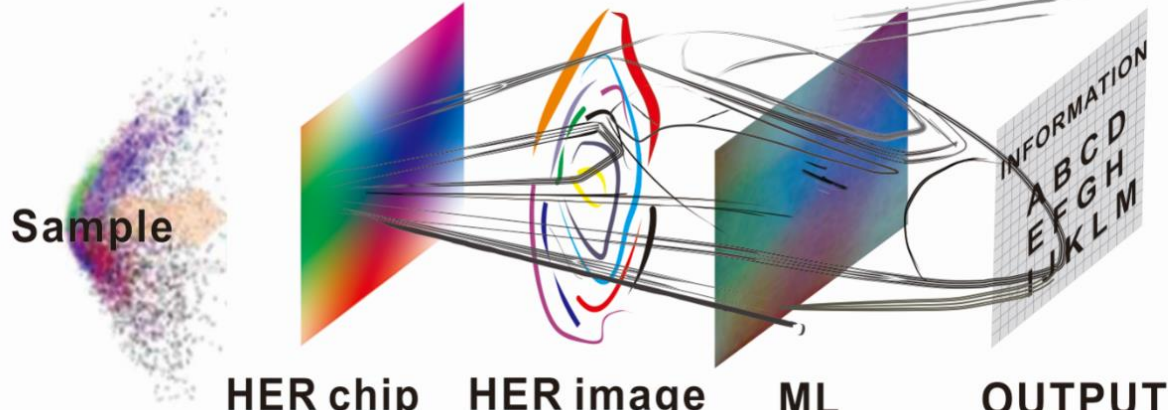

HER chip HER image

$M L$

\section{OUTPUT}

Abstract Graphic: A highway between toxicity perception and machine learning (ML). In a built highway, a sample was imaged by numerous enzyme reactions on a high-throughput chip (HER chip); and its information on acute toxicity was rapidly perceived on a trained CNN model. Here, the simple and effective HER chip just likes a high-speed rail for transportation of data between matter and ML.

Address correspondence to Jianchao Lee, Department of Environment Science, Shaanxi Normal University, Xi'an 710062, China. Tel.: +86 2985310525 Fax: +86 2985310526 . E-mail addresses: jianchaolee@snnu.edu.cn 


\section{Introduction}

The relationships between things are the important objects of natural science research. Researchers have created various research methods and means to reveal and quantify these relationships (1-3). After hundreds of years of development, it seems that we have built a powerful knowledge system. While we also found that most of the system is apt to describe the direct relations in some very simple phenomena. There is a primary fact that most natural processes are controlled by the interactions of numerous factors. Some typical examples are the reaction effects caused by the complex relationships, such as environment pollutions $(4,5)$, health risk assessment (6), catalytic effects (7), toxicity (8), etc. Therefore, the exploration of these complex relationships plays a decisive role in accurately grasping many natural laws in the future. It is also the inevitable development direction of natural science. At present, there are not many strategies for scientists in this direction, and particularly are those being adopted by ordinary researchers conveniently.

In recent years, machine learning (ML) has been developing rapidly. In many areas including business and society management, the ML application has achieved a lot of meaningful innovation (9-11). This is due mainly to some distinctive capabilities of ML, e.g., the outstanding performance on handling complex systems that are hardly tamed on the base of existing knowledge (12-14). Most reaction effects are typically complex processes, and we may use ML to explore the relationship between them.

In the course of understanding complex systems, the algorithm of ML, especially deep learning (DL), mainly executes supervised training (15). The basic strategy is to collect and obtain a big database that describes the research object, then to build a neural network consisting of numerous parameters (generally $>10^{7}$ ), later to raise the model with training on the obtained data $(15,16)$. In this process, the acquisition of a large amount of data is a key step. In today's torrent of artificial intelligence, there has been a good breakthrough in the algorithms and hardware for this kind of research. However, most of traditional experiment technologies are costly and low-efficient in the acquisition of required big data. Therefore, the method of efficiently obtaining the data of the research objects will be very important to implement the ML strategy. Even researchers need further to break through the understanding of the concept of scientific data. And be aware that the data used for ML may be quite different from the traditional scientific data, although those two kinds of data may all come from the same research object.

To obtain the data for the application of ML, there are some new technologies being improved or created in the future work. High-throughput experimentation (HTE) seems to be one of the best ways to meet the demand. The HTE, appearing in its powerful abilities in parallel operations and data production, is being adopted by some researchers (17). But we also see that today's HTE technologies are developing towards the expensive and the specialized, so that its recognition to most scientists is far less than that of the traditional experimental technologies. Moreover, current HTE technologies are always pursuing the miniaturization and parallelization of the routine experiments. It is very difficult to surpass the original understanding of the research objects due to the same perspective. Hence when acting on the ML, most HTE techniques just have to improve its tricks even principles.

One of smart HTE technologies is ink-jet printing technology (IJP) (18). The IJP usually uses the CMYK model (referring to cyan, magenta, yellow and black) to combine different chemical factors by regulating the accurate output of each ink (19). Viewing from a point of chemical operations, we can state that the essence of the IJP is a super-efficient operation to fulfill the transport of reactants. Relying on the IJP, plenty of parallel reactions will be established on a planar carrier by precise positioning of chemical or biological substances, which will generate a large amount of reaction data in a moment $(18,19)$. Sophisticated IJP experiments have great potential to meet the data needs.

Following the above exploration strategy, some specific cases will be implemented to evaluate its performance. Considering experimental conditions and technical reserves of our laboratory in recent years, we will try to explore the relationship between acute toxicity (Ac-tox) and enzyme reaction, and verify the effectiveness of the ML approach.

The Ac-tox is an important factor in comprehensive evaluation in many areas including environment, food, medicine, biology, etc. (8). Currently, general Ac-tox tests almost involve biological indicators, including fish (20), mites (21), algae (22), microorganisms (23) or other biomarkers (24). The overall experiments follow a route containing many tedious operations of continuous culture and analysis. When evaluating some samples with complex composition, researchers often select some typical toxicants (e.g. mercuric chloride, 3,5-Dichlorophenol, etc.) as reference substances to reflect the bio-toxicity indirectly (25). These 
traditional methods usually involve excessive costs in terms of instruments and time, and also hinder the presentation of Ac-tox test in many cases.

In the testing techniques of Ac-tox, LBAT test (luminescent bacteria acute toxicity test) is a smart and typical one. This technique is highly sensitive to most toxicants in a short running time. And that, the enzyme action and the chemical condition in the test are also similar to that in many higher mammals $(4,5)$. In a LBAT test (25), a sample was mixed completely with the diluent of luminescent bacteria. The intracellular luciferase (Luc) in bacteria was damaged by toxicants, and the bioluminescence was inhibited. Afterwards, the Ac-tox was assessed by luminescent inhibition rate (LIR), referring to the intensity variation in bioluminescence before and after being exposure to toxicants.

In the following work, we will try to build a simple and effective HTE bridge between Ac-tox test and ML. The bridge, grasping the common properties of the data supplied from the HTE tests and that applied in the ML algorithm, is capable of providing Ac-tox data to ML modelling quickly and massively. As emphasized, the success of this work depends mainly on the following two points, how to operate a large quantity of identifiable reactions, and how to build a proper ML model to apply these reacting data. Below, we will elaborate more on the practices of our strategy.

\section{Results \\ Overall scheme}

In the experiment work of this paper, we focused on how to fast acquire the Ac-tox dataset, and also tried to construct a suitable ML model to predict the Ac-tox. The particulars are as follows.

At first, following IJP technology, a kind of high-throughput enzyme reaction chip (HER chip, Fig. 1A) was prepared on a modified inkjet printer (Fig. S1). On the prepared chip, there are numerous varied micro-reaction dots containing accurate default of 6 enzymes or chemicals (listed in Table S1), including lead acetate, sodium citrate, glucose, glucose oxidase (GOD), catalase (POD) and 2, 6-dichlorophenol Indophenol sodium (DCIP, a blue-violet azo dye). On a reaction dot, these reagents join in a given enzymatic process that mainly refers to a glucose-oxidized reaction. The pivotal conversion is the oxidation of glucose to form oxygen radical [O] in catalyzing of GOD and POD, and the acceleration of $[\mathrm{O}]$ on the decoloration of DCIP. In the process of the chip preparation, we used a designed template $(500 \times 500 \mathrm{dpi}$, Fig. S2A) to conduct the printer to distribute accurately reactant liquids on a selected PVC-substrate. Following air-drying in room conditions, we obtained some chips consisting of about $2.5 \times 10^{5}$ reacting dots.

When the chips were used for testing, the tested sample, as a solution, was uniformly dispersed onto the chip; and then, the enzyme processes in each dot on the chip would be activated or inhibited in varying degrees. The reaction results were visually exhibited by the discoloration of DCIP. Taken as a whole, a richly-colored image (named HER image, Fig. 1A) emerged on the HER chip. Due to the massive amount of the toxicological response results on the chip the HER images recorded the Ac-tox information of the tested sample in an inapprehensible style.

For collecting a sufficient number of HER images for the later ML, we selected 4 typical toxicants as original candidates for ink solutions $\left(\mathrm{HgCl}_{2}, \mathrm{PbNO}_{3}\right.$, Tet and $\mathrm{SM} 2-\mathrm{Na}$, Table $\left.\mathrm{S} 2\right)$. All thousands of distinctive toxic samples were produced with mixing precisely the fours in IJP. This mixing process, also including the enzyme reaction, actually takes place on the HER chip efficiently, and the results displayed on the HER images.

What's more, we also directly collected Ac-tox data of all toxic samples via a modified LBAT test (Fig. 1B). The basic operation of this approach was to integrate the bacteria onto hardware platforms, which was inspired by existing luminescent sensors (26). The Ac-tox reaction between the sample and luminescent bacteria was realized by another IJP operation. Here, toxic samples as inks were printed regularly on several nutrient plates for culturing luminescent bacteria uniformly. And the change of the luminescence intensity (FI) on the plates was recorded by continuous photographing. In the end, the calculated LIRs (ranging from 0 to 1) as Ac-tox data were provided for the next ML section.

Eventually, a convolutional neural network model (15) (CNN, Fig. 1C) was adopted to perform regression analysis between the achieved HER images and LIR data. The learnable parameters of the model were iteratively adjusted by an error-backpropagation algorithm. The performance of the trained CNN was tested by using new HER images (test set) as the input to the network. The predicted LIRs were compared with the 
factual ones to estimate prediction accuracy.

\section{Producing HER images}

Preparing a greater quantity of HER chips is a foundation for building the ML dataset. We thoughtfully designed a combined template composed of 20×20 HER chips (Fig. 2SB). Under the guidance of this template, a batch of 400 HER chips was produced in an IJP cycle. After 4 cycles, 1600 HER chips (ie., $4 \times 400)$ were obtained.

On these chips, how to make them accurately and rapidly react with the test samples was also a technical challenge. In such situations, the IJP, as an efficient means of distributing material, still was a good choice. In details, we designed a sample template (Fig. 3A) that contained 400 individual recipe units (arranged as a $20 \times 20$ matrix) consisted of three $\mathrm{C} / \mathrm{M} / \mathrm{Y}$ layers. The intensity of these three colors was directly related to the concentration of 'inks' in a unit. When loading several toxicant solutions in separated cartridges on a printer (assembled rules see in Table S3), it is accessible to create larger numbers of different test toxicants (ratios listed in Table S9) on all specified units with the guidance of the template software. When these units are precisely set to 1600 chips (Fig. S3B), we got the same number of HER images (Fig. S4 and Table S5) followed performing a large batch of reactions and scanning them.

The richness and diversity of the information on HER chip are worth investigating. In Fig. 2A, the pattern on a HER chip changed before and after reacting, which was attributed mainly to all the differences of abundant reaction dots on the chip. As to the reaction course on dots (Fig. 2B), the GOD and POD were inactivated by $\mathrm{Pb}^{2+}$ and $\mathrm{Hg}^{2+}$, and then the fading process of the dye (DCIP) was inhibited. This process may be tuned by pre-existing substances on HER chips (such as Na-Citrate, Tet, SM2-Na), or others in the samples. Then bring out a result of the diverse enzyme reactions.

The information in HER images involves at least three aspects. The first is the distribution of pattern and color, which is unique for a given test sample. The second is derived from the specific chemicals at any dots of a HER chip. When we got a HER image, the specific chemical conditions will clarify quite a few temper of the enzymatic reaction. The third kind of information grows out of the cluster of numerous HER images, which is capable of connecting magically many seemingly-unrelated factors. When an image was digital (Fig.3C), these kinds of information are exhibited as 3 color dimensions (RGB, Red, Green, Blue) of pixels. And each dimension is quantified in a range of 0 to 255 ( 0 corresponds to black, 255 to white). These RGB values indirectly reflected the toxicity characteristics of test samples, which are the data basis for the later ML modelling.

\section{Collection of LIR data}

Although the LBAT test usually more available than others, it is still a time-consumed and costly operation, especially for testing thousands of samples. Thus, rapidly getting the Ac-tox data is important for us to enhance the efficiency of data acquisition.

In this case, we once again introduced the IJP tool into the improvement of the LBAT test. For some steps, we took advantage of its ability to transfer matter efficiently. In practice, before toxic reacting, the luminescent bacteria was prepared into a bio-ink and evenly printed on 4 plates (Fig. S5A). The medium was covered with bacteria uniformly released blue-green light at $450-490 \mathrm{~nm}$ in the dark, forming bioluminescence substrates. After the toxics directly act on the substrate, the luminescence process might be inhibited, and the FI variation was visually recorded on the plates. Here, by adopting the same toxic combination with Table S3, the given 1600 samples were printed on 4 bio-substrates following the layout of another template (Fig. S3A). To accurately record the fluorescence changes on whole substrates, they were immediately placed in a light-proof incubator for taking continuous pictures (Fig. S5B). Ultimately, the Ac-tox of all samples would be evaluated by the brightness variation of the collected luminescent photos (Fig. 3).

For toxicity assessment, we refer to an existed standard of luminescent bacteria tests (25). By analogy with the calculation in the standard, the LIR was calculated by the variance rate of the gray level at each reacting cells throughout the photos. Besides, for optimizing an appropriate reaction time for the Ac-tox evaluation, an experiment referring only to the 4 toxicants $\left(\mathrm{HgCl}_{2}, \mathrm{PbNO}_{3}\right.$, Tet or SM2-Na) was carried out. And detailly, they were in sequence printed and cultured according to own templates (Fig. S6), and implemented a full coverage from 0 to $100 \%$ of concentration. By observing the gray evolution of these 
4 strips, we found their gray no more changed within 5 to $30 \mathrm{~min}$. This indicated that the Ac-tox actions of the toxicants on the luminescent bacteria completed within $5 \mathrm{~min}$. Here, we chose the photos of the reaction time of 5, 15 and $30 \mathrm{~min}$ to calculate the data of the LIR (see Method section for calculation details), and listed them in Table S9.

\section{Prediction of Ac-tox in CNN}

A CNN model based on regression calculations (see in Table S7) was used to predict the Ac-tox. Selecting $\mathrm{CNN}$ as a ML model is compatible with the obtained data in image style. We found that CNN is good at extracting local features of images, and capturing the difference of these features in RGB values sensitively (10). Correspondingly, CNN possesses the potential for exploring the non-linear relation between LIR and HER images. In the CNN structure used in this study, the key functional blocks of machine learning are convolution layers and below nonlinear activation function.

$$
\left\{\begin{array}{l}
y_{i}=\sum_{j} w_{i, j} x_{j}+b_{i} \\
y_{i}^{\prime}=\max \left(y_{i}, 0\right)
\end{array}\right.
$$

Where $x$ and $y$ are input and output vectors, respectively; and $w$ and $b$ are synaptic weights and biases, respectively (11). Where $y_{i}^{\prime}$ is an activation function (ReLU) for neurons in the hidden layer of the network. The ReLU could avoid "gradient disappearing" during the computing processes (27). The principle of training a traditional network is to continuously optimize the network in $w$ (and $b)(11)$; this means a lot of training time in learning parameters. However, $\mathrm{CNN}$ can reduce the parameters in the training process through sharing weight and shrinking convolution kernels(15). The error-back-propagation algorithm can match the prediction value and factual value to the closest degree $(11,28)$. And the trained framework is capable to avoid over-fitting problem by introducing dropout layers (reducing the dimension via eliminating the relevant parameters) and regularization layers $(10,28)$.

At training stage of the CNN, the previously-acquired HER images and LIR data were served as a dataset. All the HRE images (GRB format) were adjusted to a size of $100 \times 100$ dpi and uniformly deducted background values. In Fig.4, when a HER image is taken as the input, the network first processes the images through multi-rounds of convolution, ReLU nonlinearity, and max pooling layers. And then the predicted LIR value (regularized to 0 to 1 ) is output through a fully-connected layer.

The predictive accuracy for LIR was evaluated using root mean squared error (RMSE) and the coefficient of determination $\left(\mathrm{R}^{2}\right)$ of test set via linear regression. As shown in Fig. 5A, the RMSE is $7.5 \%$ and the $\mathrm{R}^{2}$ value is 0.9203 without overfitting, which means we obtained a satisfying result on the regression of the HER images and the LIRs. A confusion matrix for classification (5 levels, Fig. 5B) is drawn based on the prediction results of the test set. Each element $(i, j)$ of the matrix represents the probability of predicting class $j$ given that the true Ac-tox class $i$ (Table S9). The accuracy rate for the classes of median-toxic, severe-toxic, and highly-toxic reached 87.6, 73.7, and 93.9\%, respectively; but only 4.5 and $56.5 \%$ of the predictive accuracy rate for the classes of non-toxic and low-toxicity. This may be due to the small number of the training data in these two levels limiting the performance of the CNN. Additionally, we randomly selected 50 new samples to verify the prediction accuracy on a small set (Fig.6). It can be found that only 6 out of 50 samples were predicted to outliers (prediction levels deviate from the real ones), and that the biases are only one level.

\section{Discussion}

The execution of our experiments benefited from the introduction of the IJP technology to prepare the high-density HER chips. Millions of independent micro-reactions were constructed easily by using a precise combination of reagents on a two-dimensional plane. Over the years, we have been bending ourselves to explore this technology, named chemical neural network chip (ChNN) in our lab habitually. On the other hand, in the field of analytical chemistry, the synthesis of numerous color (or fluorescence) indicators have accumulated lots of knowledge and products. They are contributing a treasure trove of creating many novel HTE detectors. Therefore, IJP and indicators inspire us to stare other biochemical effects. For example, Brady's team reported in Nature (Sep., 2017) that certain compounds produced by the intestinal flora had 
potential physiologic role in humans (29). Following our experimentation to combine $10^{8}$ magnitude of nutrient conditions, a proper HTE chip for surveying intestinal floras will promptly expand the searching space and simplify the experimental means. In addition, there are many important reaction processes in the field of environment or medicine. We can also build large-scale combinatorial systems for exploring huge space of more reactant types and conditions. This maybe accelerates us make new interpretations for the biological and chemical reaction relying on a large effect database.

At the same time, we are also thinking: what is the implicit link between HER images and the toxicological effects? This link may be derived from their commonality in the inhibition of the enzyme. The commonality is usually so complex that, to date researchers hardly find specific mechanisms to accurately describe it. However, we find increasingly that a neural network with millions of nodes may be a good describer of a complex effect. In other words, we can digitally describe a complex chemical process using a network with sufficient depth and breadth. Whereas, to successfully train this huge network, a satisfactory data set is necessary. Here, researchers can refer to the data amount of this work, about $1.6 \times 10^{11}$ points, (ie., $10^{8}$ points $\times 1600$ chips). Practically, the tech-nodus for introducing ML in some natural science often lies in how to gather sufficient datasets.

Here, we selected only 4 typical toxicants and their mixtures to build an Ac-tox database. Choosing such a small-scale dataset is more conform to the focus of current work, verifying the feasibility of the proposed strategy and experimentation. And while conducting constructions of many new devices and algorithms, a reasonable exploration in a small and complete data space will be affordable for us in terms of time cost. Obviously, the experimental operation of this study did not limit the variety of substances to be explored. It is foreseeable that mixtures with more-complicated components will also follow this pathway smoothly. For instance, beverages, drugs, urine, and they are the ordinaries in natural science.

What is worth mentioning, the obtained HER images pose formatted patterns following the precise control of the conditions at each dot on the chip. This is obviously different from usual image datasets supplying ML, such as these pictures of cancer pathology, fundus scans, or street view. These databases often contain at least thousands to millions of pictures, which are much larger than the training set in our job. But it needs to be pointed out that the amount of effective information in these images is too low. One vivid description is that the ordinary images are similar to some stone bombs collected from various places, but well-conceived images are modern standard ammunitions. For the "ML cannon", their efficiency will be very different.

In this study, lots of bio-effect data were easily produced by HER chips. But we can say humorously that these data are "useless". That is, we hardly extract directly the toxicological info from the HER images only with the existing knowledge system. Nevertheless, it is because we do not pursue "common-sense understanding and application" of data that allows us to focus more on creating cost-effective and efficient ways of data acquisition. This starting point may also be an appropriate strategy for machine learning to explore various scientific issues in the future. Because there are still too few databases that are directly understood by human and meet the appetite of ML.

Briefly, we have initially attempted to establish machine learning approaches of exploring the relationships among complex reaction processes. And adopting a smart experimentation of luminescent bacteria, we demonstrated the validity and efficiency of CNN modelling. The key to this approach is creating powerful means to actively produce mass of new data to describe the research objects. Also, the introduction of ML may provide other researchers a new thought for the study of other complex reaction effects.

\section{Materials and Methods}

All of the chemical reagents listed in Table S1-2 were (analytical grade, $\geq 99 \%$ ) purchased from Aladdin Sigma-Aldrich. Photobacterium phosphoreum (T3 spp.) was purchased from Nanjing Institute of Soil Science. A white PVC plate (A4, Guangzhou Chongze Tech. Co.) was used as the printing substrate. A commercial ink-jet printer (R230 printer, Epson Company) was reformed. An image scanner (S48) was purchased from Plustek Technology Co., Ltd., China. A high-sensitivity CCD camera (A75, Canon Company) that can be controlled by a computer was used to record FI variations of luminescent bacteria.

\section{Reform of ink-jet printer}


A commercial ink-jet printer was reformed. It has the advantages of high accuracy, multiple channels, non-contact, and no heating (ensuring bio-ink activity). As shown in Fig. S1, the paper entry mechanism of the printer was modified as a fixed-platform $\mathbf{1}$ and it has a positioning slot $\mathbf{3}$ that can fix the substrates. Then, the printer's driveline was modified. That is, the printing head $\mathbf{6}$ is reciprocated along a horizontal guide rail 5 under the control of an additional stepping motor 7, while it can also advance along a vertical guide rail 4 at a constant speed. Additionally, the printing head has six cartridges refer to color channels of C/M/Y. When working, the cartridges are emptied to be applied to fill solutions containing enzymes or chemicals (Table $\mathrm{S}$ 1 to 2$)$.

\section{Producing HER images \\ Preparation of printing solutions}

For preparing the HER chip, 6 solutions containing enzymes or chemicals were adopted, including $\left(\mathrm{CH}_{3} \mathrm{COO}\right)_{2} \mathrm{~Pb}$, sodium citrate, POD ( $\geq 300$ units/mg), GOx ( $\geq 100$ units/mg), Glucose and DCIP (see in Table S1). A series of precursor solutions were prepared in given concentrations respectively. Particularly, the surface tension of the inks is adjusted to ensure the normal operation of the printer. Here, ethylene glycol monobutyl (DGBE) was added to lead acetate, sodium citrate, glucose, and DCIP, respectively. For the preparation of GOD or POD ink, PEG-20000 and tert-butanol were added to the enzyme solution $(1 \mathrm{mg} / \mathrm{mL}$ of protein) in phosphate buffer $(\mathrm{pH} 7.5,50 \mathrm{mM})(19)$. All inks were adjusted to exactly match the original ink $\left(\sim 45 \mathrm{dyn} \mathrm{mL}^{-1}\right)$.

\section{Design of HER-Chip template}

In Fig. S2A, single HER-chip template is designed as a rectangle $(500 \times 500$ dpi) composed of 6 color layers, in which CMY refers to three inks: regular cyan, magenta, and yellow. According to the color distribution (pre-set CMY values), layers 1-3 indicates that the output of each ink ranged 0-100\% in steps of 1\%, while the inks on layers 4-6 are in a constant output of 100\%. All the layers were corresponded to the enzymes (or chemicals) as listed in Table S1. At last, this CMY model will generate a color space theoretically containing $2.5 \times 10^{5}$ dots by various combinations of these layers. Besides, a composition template containing 400 HER chips also was designed in Fig. S2B. The chips are spatially arranged in a $20 \times 20$ matrix, and each one is made up layers 1-6. By printing 4 pages (one page 400 chips), 1600 HER chips were parallelly prepared.

\section{Design of sample template}

A sample template (Fig. S3a) contained 20×20 individual recipe units was designed, and each unit $(10.0 \times 10.0 \mathrm{~mm})$ represents a mixed sample of 3 toxic compounds (selected from Table S2). All CMY layers in the template respectively contain a subtle linear-gradient of color (ranged from 0-100\%) in different directions. By arranging multiple inks in the template (assembled rules see Table S3), 1600 mixed-samples were formed and printed.

\section{Preparation of HER chip}

In a typical step, the cartridges were washed with distilled water and ethanol, and 6 inks (listed in Table S1) were added to the cartridges. The selected PVC substrate was placed smoothly on 1, and fixed at a designated location. During printing, the nozzles were guided by the composition template (Fig. S2B) to print the inks on the substrate. Specially, by stacking the layers, 6 inks in Table S1 were combined along certain distribution directions and quantities. For in-situ mixing of the reactants on the HER chip, the obtained HER chips were sprayed with pure water in an ultrasonic actuator (droplets with size of about 3 to $5 \mu \mathrm{m}$ ) followed by air-drying. Then, all the resulting chips were placed in an airtight vessel with deoxidant in the dark.

\section{AC-tox reaction between HER chip and samples}

All the Ac-tox reactions between the HER chip and sample were carried out by positioning overprinting (shown in Fig. S3B). Before reacting, 4 toxic compounds $\left(\mathrm{HgCl}_{2}, \mathrm{PbNO}_{3}\right.$, Tet and SM2-Na, listed in Table S2) were prepared into a series of solutions (in $2 \% \mathrm{NaCl}$ ), and added to the cartridges as original inks. Meanwhile, one of the prepared substrate comprised of 400 HER chips was placed and fixed on 1. During printing, the 4 toxicant solutions as ink were distributed accurately following the guidance of the template in Fig. S3A. Wait for the chip to dry (wait about 30 seconds), the 1600 HER images (see in Fig. S4) are accurately scanned by a commercial laser scanner.

\section{Collection of AC-tox data}




\section{Preparation of nutrient plates}

A classically formulated Sea Water Broth (SWB) culture medium was prepared as the nutrient plate. SWB contained: yeast extract, $2.5 \mathrm{~g}$; tryptone, $2.5 \mathrm{~g} ; \mathrm{Na}_{2} \mathrm{HPO}_{4} \cdot 12 \mathrm{H}_{2} \mathrm{O}, 6.3 \mathrm{~g} ; \mathrm{KH}_{2} \mathrm{PO}_{4}, 0.5 \mathrm{~g} ; \mathrm{NaCl}, 15 \mathrm{~g}$; agar, $10 \mathrm{~g}$ and $\mathrm{pH}$ 6.8-7.2 in $400 \mathrm{~mL}$ distilled water. The obtained mixed suspension was sterilized in a sterilizer at $394 \mathrm{~K}$ for 20 minutes, and then was evenly distributed to four rectangular glass dishes $(10 \times 10 \mathrm{~cm})$, cooling to room temperature. Then, these obtained nutrient plates were preserved in a sterilizer box for later use.

\section{Preparation of luminescent bacteria diluent}

Freshly prepared Photobacterium phosphoreum (T3 spp.) was quantitatively inoculated into a SWB medium ( $200 \mathrm{~mL}$, without agar), and then it oscillated for $14 \mathrm{~h}$ at $293 \mathrm{~K}$ in a constant temperature shaker for further replication. Then, the reconstituted bacterial suspension $\left(1.0 \times 10^{8}\right.$ cells $\mathrm{mL}^{-1}$ luminous bacteria) was diluted (10:1) as a bio-ink for the latter LBAT test.

\section{Modified LBAT test}

In preparation, four prepared $10 \times 10 \mathrm{~cm}$ nutrient plates were placed on the designated areas of a sterile glass plate, and $5 \mathrm{~mL}$ of bio-ink was added to the cartridge. Then, the bio-ink was uniformly printed on the plates forming bio-substrates, and wait for 4 hours to make the bacteria to light steadily.

Next, the toxicity test still was applied on above substrates (see in Fig. S5). Four sample templates are arranged parallelly on these substrates, each substrate corresponds to $20 \times 20$ samples. According to the assembled rules in Table S3, aforementioned $\mathrm{HgCl}_{2}$ etc. toxic solutions (Table S2) as original inks once again were respectively printed on the substrates, appearing 1600 reacting cells. Then, a high-sensitivity CCD camera was used for continuous photographing (One shot every 1 minute, continuous shooting 12 hours) and a series of bio-luminescence photos (Fig. 3) were acquired. Shooting parameters see in Table S6. In addition, a blank control experiment on regions without printing toxicants was conducted simultaneously.

\section{LIR Calculation}

Average gray values of all the reacting cells on pictures in Fig. 3 were extracted using Matlab. Variance rate of gray level on the same cell at various times $(t=0,5,15,30 \mathrm{~min})$ was used to represent the LIR of the sample. The calculation process as follows:

Calculate the luminous intensity correction factor $\left(\mathrm{f}_{\mathrm{kt}}\right.$-value) from the measured gray value using Equation (1). This factor is used to correct the initial values $I_{0}$ of all the samples before they are used as reference values. It can be used to correct the luminescence intensity change of CK regions (only luminescent bacteria without printing the samples) due to the evaporation of water from the medium.

$$
\mathrm{f}_{\mathrm{kt}}=\frac{\mathrm{G}_{\mathrm{kt}}}{\mathrm{G}_{0}}
$$

Where $f_{k t}$ is the luminous intensity correction factor, $G_{k t}$ is the average gray value of CK regions at $t=5$, 15 or $30 \mathrm{~min}, \mathrm{G}_{0}$ is the average gray value of the $\mathrm{CK}$ region at $\mathrm{t}=0 \mathrm{~min}$.

$$
\mathrm{G}_{\mathrm{ct}}=\mathrm{f}_{\mathrm{kt}} \times \mathrm{G}_{0}
$$

Where $\mathrm{G}_{\mathrm{ct}}$ is the corrected value of $\mathrm{G}_{0}$ before the addition of the test sample.

The LIR using Equation (3):

$$
\mathrm{LIR} \%=\frac{\mathrm{G}_{\mathrm{Ct}}-\mathrm{G}_{\mathrm{t}}}{\mathrm{G}_{\mathrm{Ct}}} \times 100 \%
$$

$\mathrm{G}_{\mathrm{t}}$ is the average gray value at $\mathrm{t}=5,15$ or 30 min after the Ac-tox reaction. LIR is luminescent inhibition rate of one of the samples. Here, at different shooting time pictures, the average gray values of the same samples were extracted from the same reacting cells. Similarly, LIRs of other samples were calculated by the same way. And finally only the data of $\mathrm{t}=15 \mathrm{~min}$ were used as the label for HER images. 


\section{Supplementary Materials}

Fig. S1. The reformed ink-jet printer.

Fig. S2. Design of printing templates.

Fig. S3. Design of sample template; and application of the Ac-tox reactions.

Fig. S4. Obtained 1600 HER images.

Fig. S5. Collection of LIR data.

Fig. S6. Gray variations on the luminescence images.

Table S1. Configuration of inks on a single HER template Table S2 Shooting parameters.

Table S2. List of 4 toxic compounds.

Table S3. Assembled rules of 4 toxic compounds on the sample template.

Table S4. Physical properties of candidate print substrates.

Table S5. Coding numbers of 1600 HER chips (or images).

Table S6. Shooting parameters.

Table S7. Detailed architecture of CNN.

Table S8. Ac-tox evaluation standard.

Table S9. LIR data and sample composition.

Table S10. LIR data for training set $(80 \%)$.

Table S11. LIR data for test set (20\%)

Acknowledgments: Thanks to Chaoqun Zheng and Baoxin Zhai for their work in preparing the materials in the early stage. Especially, Qiannan Duan wants to thank her parents (Mr. Duan and Mrs. Wu), for their love, patience and selfless support over the years. Finally, we salute to the great physicist, Stephen William Hawking.

Funding: This work is supported by the National Natural Science Foundation of China (No.50309011) and the Scientific Research Foundation for the Returned Overseas Chinese Scholars (08501041585).

Competing interests: The authors declare no competing interests.

Data and materials availability: Additional figures, tables, and descriptions. Besides, the original HER images used for training and testing the models can be downloaded from an URL, http://yunpan.snnu.edu.cn:80/\#/link/3F8867BF29D30048668646809875892D, with a fatch code: 6Wax. All the data that support the results of this study are available from the corresponding author.

\section{References}

1. S. Allesina, J. Grilli, G. Barabas, S. Tang, J. Aljadeff, A. Maritan, Predicting the stability of large structured food webs. Nat. Commun. 6, 7842 (2015).

2. Y.-Y. Liu, Theoretical progress and practical challenges in controlling complex networks. Natl. Sci. Rev. 1, 341-343 (2014).

3. J. Ruths, D. Ruths, Control profiles of complex networks. Science 343, 1373-1376 (2014).

4. T. Wang, Y. Liu, D. Wang, Z. Lin, Q. An, C. Yin, Y. Liu, The joint effects of sulfonamides and quorum sensing inhibitors on Vibrio fischeri: Differences between the acute and chronic mixed toxicity mechanisms. J. Hazard. Mater. 310, 56-57 (2016).

5. H. Hu, C. Jiang, H. Ma, L. Ding, J. Geng, K. Xu, H. Huang, H. Ren, Removal characteristics of DON in pharmaceutical wastewater and its influence on the N-nitrosodimethylamine formation potential and acute toxicity of DOM. Water Res. 109, 114-121 (2017).

6. H. Wang, N. Wang, J. Qian, L. Hu, P. Huang, M. Su, X. Yu, C. Fu, J. Feng, Q. Zhao, Urinary antibiotics of pregnant women in Eastern China and cumulative health risk assessment. Environ. Sci. Technol 51, 3518-3525 (2017).

7. L. Ward, A. Agrawal, A. Choudhary, C. Wolverton, A general-purpose machine learning framework 
8. D. Wang, Y. Gu, M. Zheng, W. Zhang, Z. Lin, Y. Liu, A Mechanism-based QSTR Model for Acute to Chronic Toxicity Extrapolation: A Case Study of Antibiotics on Luminous Bacteria. Sci. Rep. 7, 6022 (2017).

9. D. Ahneman, Predicting reaction performance in CN cross-coupling using machine learning (vol 360, pg eaat7648, 2018). Science 360, 613-613 (2018).

10. A. Esteva, B. Kuprel, R. A. Novoa, J. Ko, S. M. Swetter, H. M. Blau, S. Thrun, Dermatologist-level classification of skin cancer with deep neural networks. Nature 542, 115-118 (2017).

11. Y. Jo, S. Park, J. Jung, J. Yoon, H. Joo, M.-h. Kim, S.-J. Kang, M. C. Choi, S. Y. Lee, Y. Park, Holographic deep learning for rapid optical screening of anthrax spores. Sci. adv. 3, e1700606 (2017).

12. R. Gómez-Bombarelli, J. N. Wei, D. Duvenaud, J. M. Hernández-Lobato, B. Sánchez-Lengeling, D. Sheberla, J. Aguilera-Iparraguirre, T. D. Hirzel, R. P. Adams, A. Aspuru-Guzik, Automatic chemical design using a data-driven continuous representation of molecules. ACS Cen. Sci. 4, 268-276 (2018).

13. Y. D. Hezaveh, L. P. Levasseur, P. J. Marshall, Fast automated analysis of strong gravitational lenses with convolutional neural networks. Nature 548, 555-557 (2017).

14. Y.-Y. Liu, J.-J. Slotine, A.-L. Barabási, Observability of complex systems. P. Natl. Acad. Sci. USA. 110, 2460-2465 (2013).

15. Y. LeCun, Y. Bengio, G. Hinton, Deep learning. nature 521, 436-444 (2015).

16. I. Goodfellow, Y. Bengio, A. Courville, Y. Bengio, Deep learning. (MIT press, Cambridge, 2016), vol. 1.

17. K. Troshin, J. F. Hartwig, Snap deconvolution: An informatics approach to high-throughput discovery of catalytic reactions. Science 357, 175-181 (2017).

18. L. Wang, J. Lee, M. Zhang, Q. Duan, J. Zhang, H. Qi, Fluorescence imaging technology (FI) for high-throughput screening of selenide-modified nano- $\mathrm{TiO}_{2}$ catalysts. Chem. Commun. 52, 2944-2947 (2016).

19. Y. Zhang, F. Lyu, J. Ge, Z. Liu, Ink-jet printing an optimal multi-enzyme system. Chem. Commun. 50, 12919-12922 (2014).

20. N. Klüver, M. König, J. Ortmann, R. Massei, A. Paschke, R. Kühne, S. Scholz, Fish embryo toxicity test: Identification of compounds with weak toxicity and analysis of behavioral effects to improve prediction of acute toxicity for neurotoxic compounds. Environ. Sci. Technol. 49, 7002-7011 (2015).

21. M. Neves et al., Biochemical and populational responses of an aquatic bioindicator species, Daphnia longispina, to a commercial formulation of a herbicide (Primextra ${ }^{\circledR}$ Gold TZ) and its active ingredient (S-metolachlor). Ecol. Indic. 53, 220-230 (2015).

22. J. Villain, L. Minguez, M.-P. Halm-Lemeille, G. Durrieu, R. Bureau, Acute toxicities of pharmaceuticals toward green algae. mode of action, biopharmaceutical drug disposition classification system and quantile regression models. Ecotoxicol. Environ. Saf. 124, 337-343 (2016).

23. S. C. Edberg, E. W. Rice, R. J. Karlin, M. J. Allen, Escherichia coli: the best biological drinking water indicator for public health protection. J. Appl. Microbiol. 88, 106S-116S (2000).

24. A. S. Thakor, R. Luong, R. Paulmurugan, F. I. Lin, P. Kempen, C. Zavaleta, P. Chu, T. F. Massoud, R. Sinclair, S. S. Gambhir, The fate and toxicity of Raman-active silica-gold nanoparticles in mice. Sci. Transl. Med. 3, 79ra33 (2011).

25. D. ISO, 11348-3 (2007) Water quality-determination of the inhibitory effect of water samples on the light emission of Vibrio fischeri (Luminescent bacteria test)-part 3: method using freeze-dried bacteria. PN-EN ISO.11348 (2007)

26. M. Mohseni, J. Abbaszadeh, S. S. Maghool, M. J. Chaichi, Heavy metals detection using biosensor cells of a novel marine luminescent bacterium Vibrio sp. MM1 isolated from the Caspian Sea. 
Ecotoxicol. Environ. Saf. 148, 555-560 (2017).

27. C. Szegedy, W. Liu, Y. Jia, P. Sermanet, S. Reed, D. Anguelov, D. Erhan, V. Vanhoucke and A. Rabinovich, Going deeper with convolutions, in proceedings of the IEEE conference on computer vision and pattern recognition (IEEE, 2015), pp. 1-9.

28. D. T. Ahneman, J. G. Estrada, S. Lin, S. D. Dreher, A. G. Doyle, Predicting reaction performance in C-N cross-coupling using machine learning. Science 360, 186-190 (2018).

29. L. J. Cohen, D. Esterhazy, S. H. Kim, C. Lemetre, R. R. Aguilar, E. A. Gordon, A. J. Pickard, J. R. Cross, A. B. Emiliano, S. M. Han, Commensal bacteria produce GPCR ligands that mimic human signaling molecules. Nature 549, 48-53 (2017). 

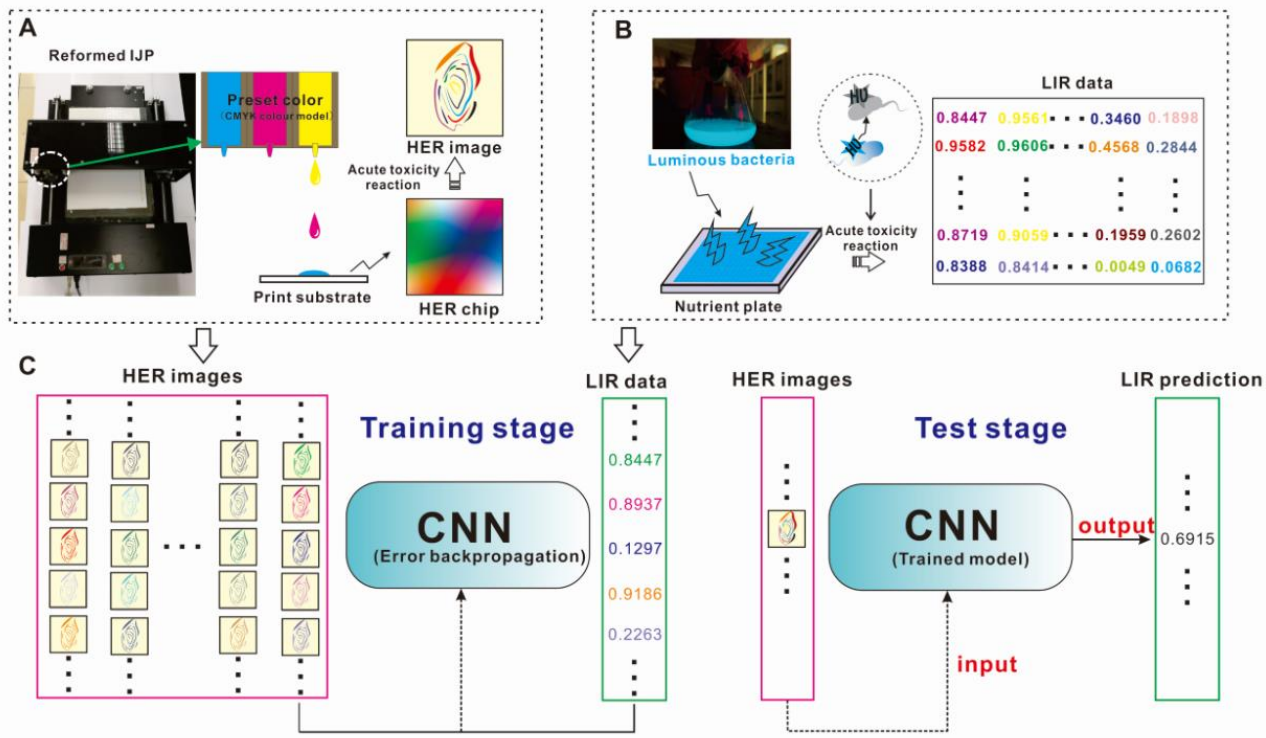

Fig. 1 Scheme for constructing a strategy between Ac-tox prediction and ML. (A) Preparation of the HER chip by IJP; and the HER image was received after the chip reacting with the test sample. (B) A modified LBAT test for collecting LIR data. (C) CNN for LIR prediction. At the training stage, the HER images and LIR data were as the training set. At the testing stage, some HER images of unknown samples as input, and their LIRs were predicted by the trained CNN. 


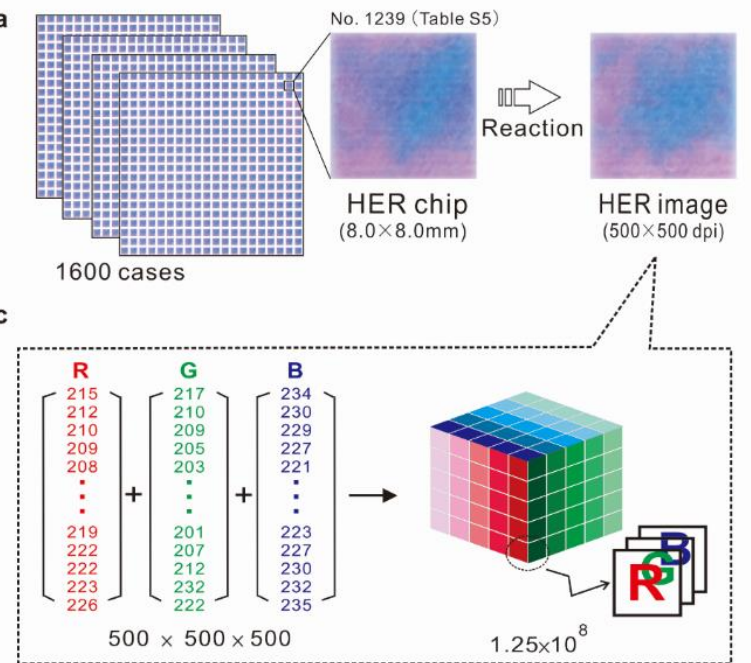

Fig. 2 (A) Obtained 1600 HER chips and appearance of a HER chip/image. (B) The enzymatic process in a dot on chip. Process 1 to 3: GOD catalyzes the oxidation of b-D-glucose into D-glucono-1, 5 lactone, and generates an equivalent amount of $\mathrm{H}_{2} \mathrm{O}_{2}$. Then, DCIP is catalyzed by POD in the presence of $\mathrm{H}_{2} \mathrm{O}_{2}$, exhibiting a dye-fading process (due to the $\mathrm{C}-\mathrm{N}$ double bond in the structure of DCIP is broken by [O]). In process 4, GOD and POD are inactivated partly by $\mathrm{Pb}^{2+}$, which inhibits the process $1-3$. While $\mathrm{Pb}^{2+}$ combined with the citrates, the $\mathrm{Pb}^{2+}$ transforms into $\mathrm{C}_{12} \mathrm{H}_{10} \mathrm{O}_{14} \mathrm{~Pb}_{3}$, and then, oppositely, 1-3 will be protected. In the meantime, the toxic compounds (TCs) in the sample maybe participate in all the process 1-5 (red dotted line), activating or inhibiting the 1-3. (C) Color dimensions of the HER image. In our experiment, the number can reach about $10^{8}$. 


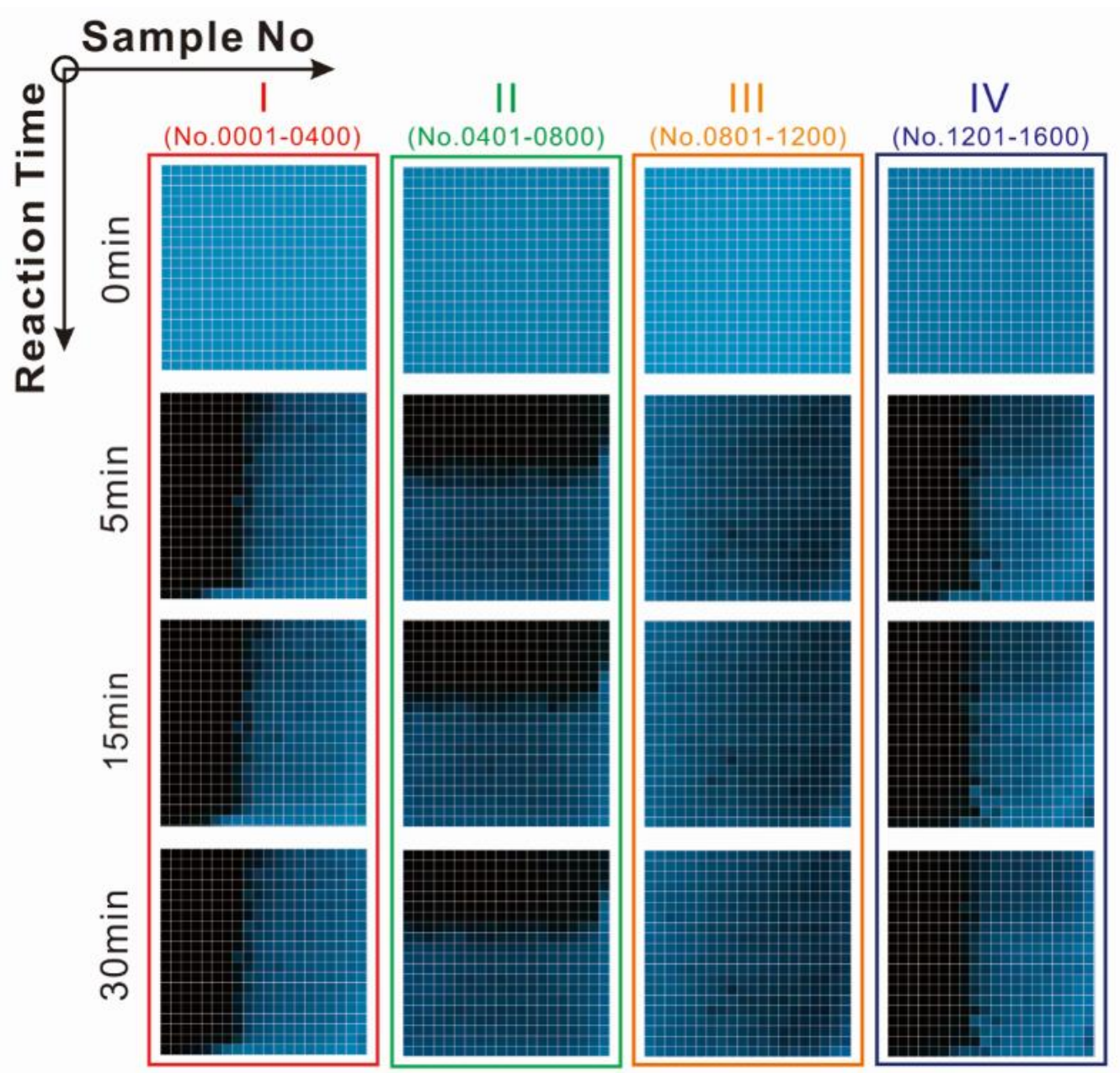

Fig. 3. Bio-luminescence photos at $\mathbf{t}=\mathbf{0}, \mathbf{5}, 15$ and 30min, respectively. The photographing parameters are listed in Table S6. 

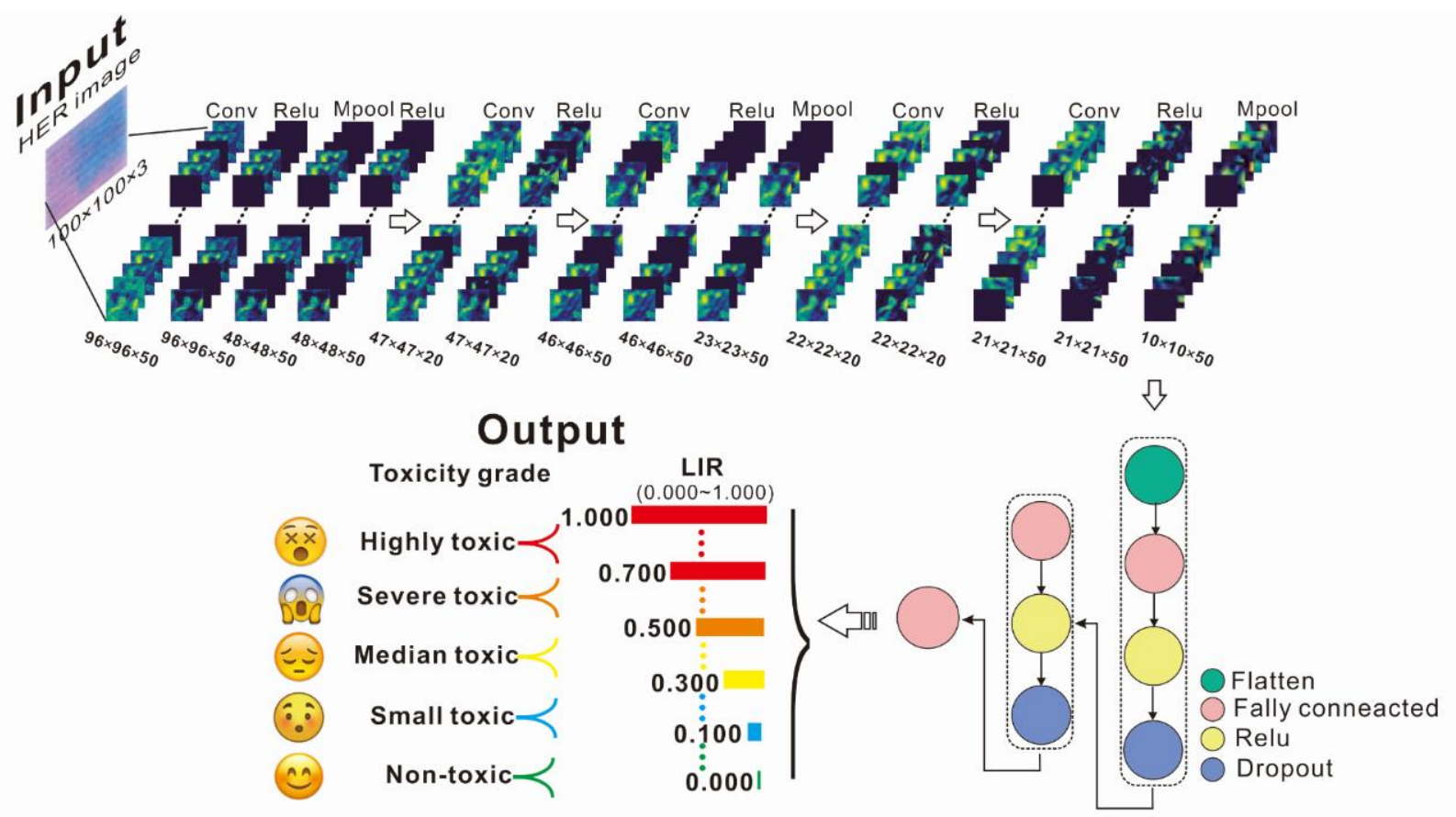

Fig. 4. CNN framework for Ac-tox prediction based on HER images. The network is a regression-based CNN architecture. Data flow is from left to right: a HER image (No.0001 as a case) is taken as the input, the network first processes the image through five rounds of hidden layer, including convolution, ReLU nonlinearity, and max pooling layers. Then, the data flow through two fully-connected, ReLU and dropout-regularization layers. The output layer will give out the predicted LIR. Meanwhile, 5 toxicity grades also are as output in this CNN framework. 

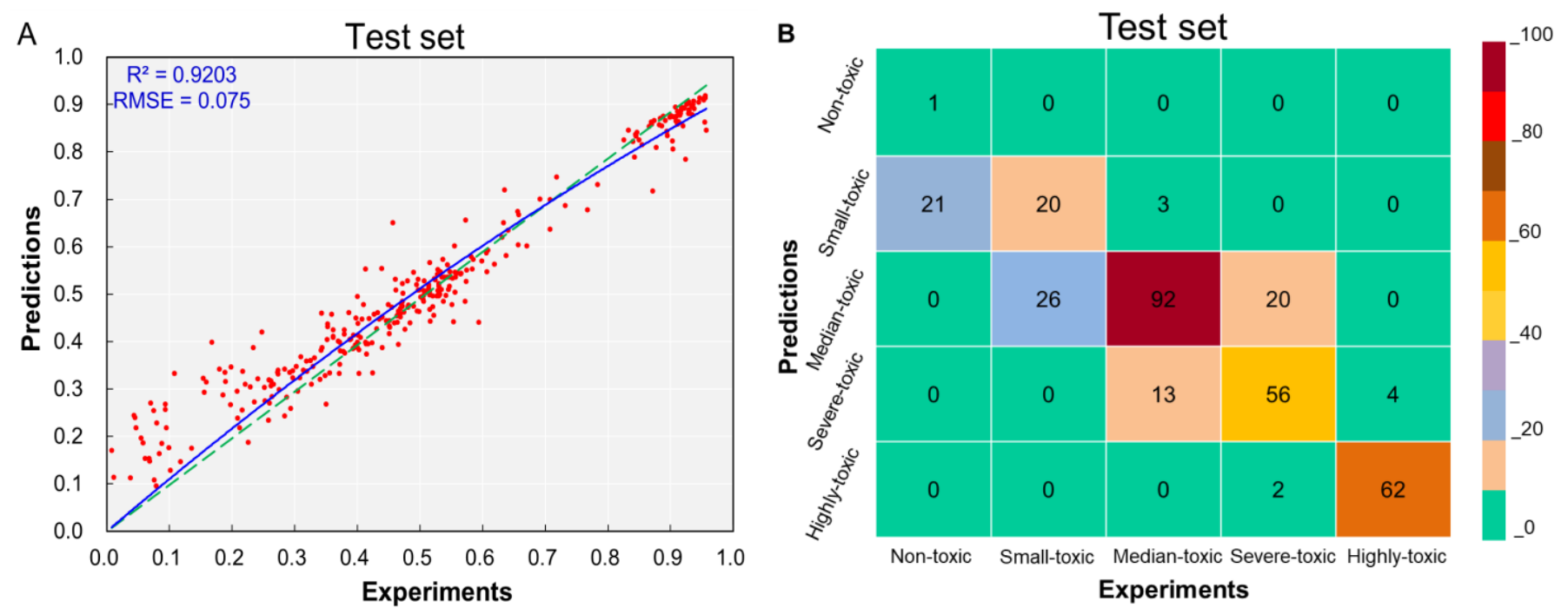

Fig. 5 Performance of the CNN framework. (A) Test set performance plots. Observed vs. predicted plots of LIR were predicted by the CNN. For the model, an 80/20 split of training and test data (Table S10-S11); and only the test set shown in plots. Superiority of the algorithm was evaluated by linear regression analysis: RMSE, root mean square error; R2, coefficient of determination; dashed line, $\mathrm{y}=\mathrm{x}$ line; solid line, Loess best-fit curves. (B) Confusion matrix illustrating the performance of the CNN. 


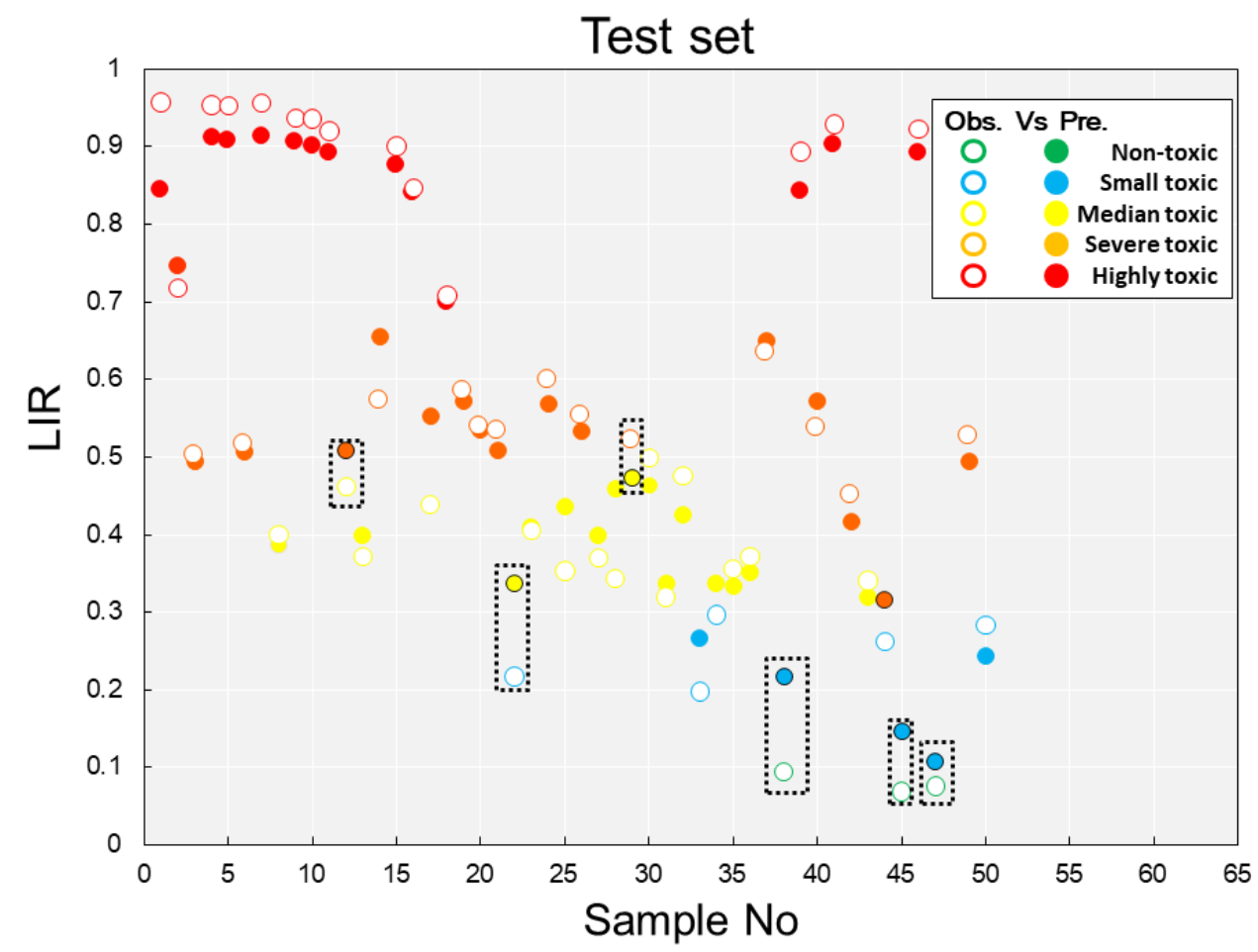

Fig. 6 An Ac-tox level prediction for 50 random samples. Solid or open dot is the predicted or observed LIR data, respectively. Predicted outliers are circled in dotted line. 